\title{
4 Methods
}

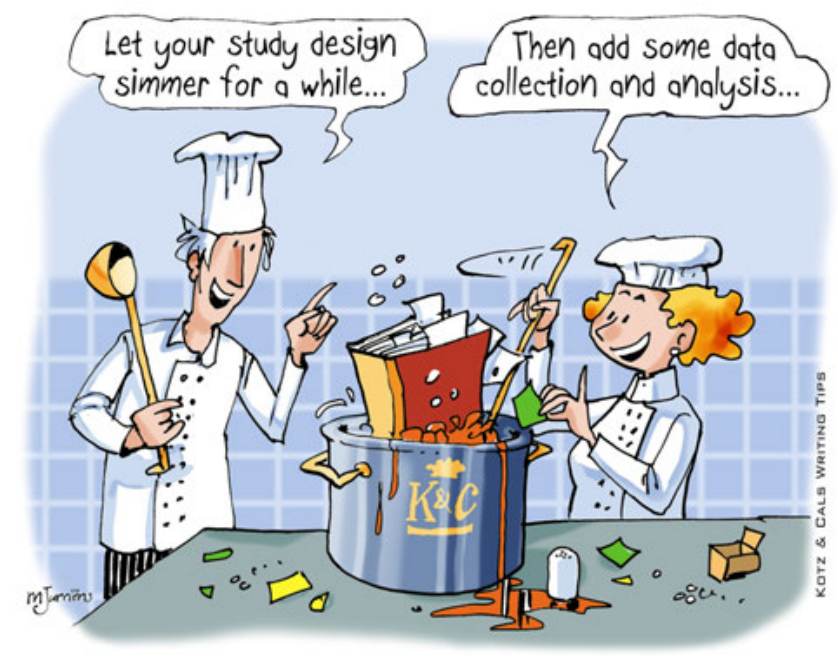

Tip 4 - Methods: provide a cookbook with the study’s ingredients!

\section{What you should know}

If you consider a research study as a delicate dish of knowledge, a paper's methods section would be like a recipe that lists all the necessary ingredients of the study and how they need to be combined during the cooking. Ideally, it allows the dish to be prepared again with the same result. The methods section ties the introduction to the results section to create a clear storyline; it should present the obvious approach to answer the research question and define the structure in which the results will be presented later.

The methods section of a paper presenting original research from a quantitative study usually has four basic elements: study design, setting and subjects, data collection, and data analysis. It is quite common to use such subheadings to structure the section (the target journal may offer specific guidance). The authority providing ethical clearance for the study needs to be stated as well, just as if the study has been registered, both with identifiers. 


\section{What you should do}

Start by developing an outline with the basic elements of the methods section (see part 1: how to get started). If available, refer to a published protocol or previously published papers from the same research project for additional information about the methods. This allows you to keep the methods section more concise. Be sure, however, to include all information that the reader needs to understand how the key findings in this paper were derived.

Mention the design of the current study, such as randomised controlled trial, prospective/retrospective cohort study, case-control study, or cross-sectional survey. If you find it difficult to fit your study into a specific type of design, try to describe the key design components, for example whether it was and interventional and/or observational study and whether data were collected longitudinally and/or cross-sectionally.

Explain when and where the study was conducted, how the sample was recruited or selected, and which inclusion/exclusion criteria were applied. Provide a sample size calculation for studies set up to statistically test a specific hypothesis.

As regards the data collection, define precisely what exposure (e. g., stressful life events) or intervention (e. g., cognitive behavioural therapy) you investigated, what outcomes you measured (e.g., depression), how you measured them (e.g., using a self-reported depression scale) and when measurements were made (e.g., during the screening visit and after 12 months of follow-up). Cite original research on existing measurement tools you used, and state if you designed a tool specifically for the study. Provide details of measurement properties (reproducibility, validity, responsiveness) if these are crucial for the interpretation of the main results. A useful order if you used various measurements is to start with the outcome measure (or dependent variable), followed by the exposure measures (or main independent variables), and possible covariates or confounders.

Match the part on data analysis with the research questions. If you present a primary research question in your introduction and one or more secondary questions, start by explaining the primary analysis, followed by the secondary analyses. Provide sufficient detail on the statistical techniques you used; do not assume that readers understand what you did only from the name of a technique. Be very clear about the definition and operationalisation of the dependent and main independent variable, the use of covariates (i.e. if and how you adjusted your analyses), and the handling of missing data. Be honest and clear about the analyses you intended a priori to test your hypothesis, and the analy- 
ses that were exploratory (post hoc). Consider providing more detailed information on the methods in the paper's web-only supplement or by using an open access online repository (such as the Open Science Framework). Avoid putting results in the methods section, such as numbers of subjects recruited and followed up.

As there may be various ways to answer a research question, try to explain, where necessary, why you made certain methodological choices and why you think these were the best options given the context. You can demonstrate the credibility of your methods by citing previous research.

After you have drafted the methods section, ask yourself: "Would a researcher be able to reproduce our study with the information I provide in this paper?" Also check whether the section contains redundant information which is not necessary to understand the paper's storyline. This check is particularly important when the paper is one of many arising from the same, larger research project. Only describe methods for which results are presented later.

\section{Checklist for the methods}

- Include basic information on study design, setting and subjects, data collection, data analysis and ethical approva

- Refer to previous publications from the same large research project, such as a study protocol, for additional information (if applicable)

- Consider providing more detailed information on the methods as web-only supplementary material or by using an open access online repository

- Ask yourself: "Would a researcher be able to reproduce the study with the information I provide in this paper?" 